\title{
Structural analysis of an InGaN/GaN based light emitting diode by $\mathrm{X}$-ray diffraction
}

\author{
M. K. Öztürk · Yu Hongbo · B. Sarıkavak • \\ S. Korçak $\cdot$ S. Özçelik $\cdot$ E. Özbay
}

Received: 3 February 2009/Accepted: 1 April 2009/Published online: 18 April 2009

(C) Springer Science+Business Media, LLC 2009

\begin{abstract}
The important structural characteristics of hexagonal $\mathrm{GaN}$ in an $\mathrm{InGaN} / \mathrm{GaN}$ multi quantum well, which was aimed to make a light emitted diode and was grown by metalorganic chemical vapor deposition on $c$-plain sapphire, are determined by using nondestructive high-resolution X-ray diffraction in detail. The distorted $\mathrm{GaN}$ layers were described as mosaic crystals characterized by vertical and lateral coherence lengths, a mean tilt, twist, screw and edge type threading dislocation densities. The rocking curves of symmetric (00.l) reflections were used to determine the tilt angle, while the twist angle was an extrapolated grown $\omega$-scan for an asymmetric (hk.l) Bragg reflection with an $h$ or $k$ nonzero. Moreover, it is an important result that the mosaic structure was analyzed from a different (10.l) crystal direction that was the angular inclined plane to the $z$-axis. The mosaic structure parameters were obtained in an approximately defined ratio depending on the inclination or polar angle of the sample.
\end{abstract}

M. K. Öztürk · S. Korçak · S. Özçelik

Department of Physics, Gazi University, 06500 Teknikokullar, Ankara, Turkey

M. K. Öztürk ( $\varangle)$

Department Mineral Analysis and Technology, MTA, 06520 Ankara, Turkey

e-mail: ozturkm@gazi.edu.tr

Y. Hongbo · E. Özbay

Nanotechnology Research Center, Department of Physics,

Department of Electrical and Electronics Engineering,

Bilkent University, 06800 Bilkent, Turkey

B. Sarıkavak

Department of Physics, Kastamonu University, Kastamonu, Turkey

\section{Introduction}

III-nitrides have attracted attention for optoelectronic device applications whose emission wavelength range from green to ultraviolet light due to their wide band gap [1]. It is well known that the high-density threading dislocations (TDs) are easily introduced in GaN buffer due to the large mismatch of the lattice constants and the significant difference of the thermal expansion coefficient between $\mathrm{GaN}$ epilayers and sapphire substrates [2]. Therefore, GaN epitaxial films are often grown as mosaic structural crystals that can be characterized by means of tilt and twist angles which are related to the high-density TDs. TDs have significant effects on the performance and lifetime of GaN-based devices [3, 4]. Since these devices have recently become available for commercial use, many groups have studied the homoepitaxial growth to enhance the reliability of optoelectronic devices by minimizing the defects or dislocation density of $\mathrm{GaN}$ epilayers [5-9].

The model of mosaic crystals is described by the structures shown in Fig. 1. The crystal layer that was formed from mosaic blocks is assumed to consist of single crystallites. Since the crystallites coherently scatter the $\mathrm{X}$-rays, their dimensions are called vertical and lateral coherence lengths. Mosaic blocks are assumed to be slightly disoriented with respect to each other. The out-ofplane rotation of the blocks parallel to the surface is the mosaic tilt, in which the in-plane rotation around the surface normal is the mosaic twist. The average absolute values of the tilt and twist are directly related with the full width at half maximum (FWHM) of the corresponding distributions of the crystallographic orientations [10]. Dislocation densities are correlated to the FWHM values and are calculated from twist angles [11]. 


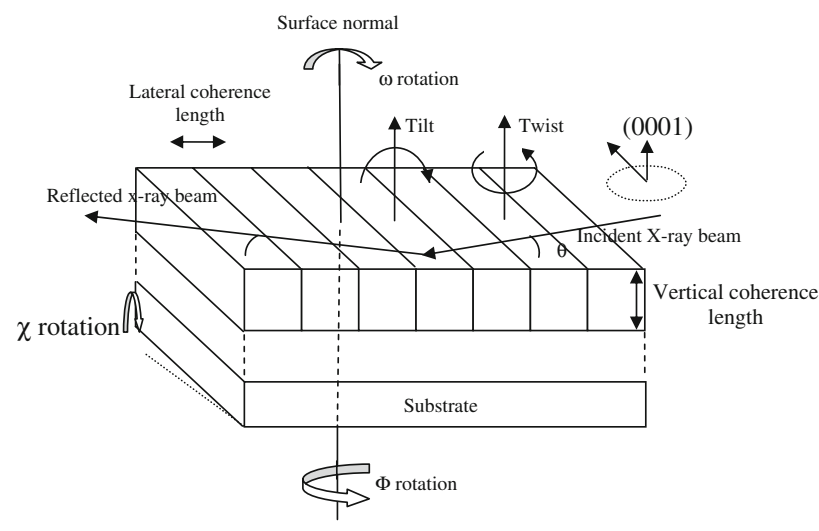

Fig. 1 Schematic diagram of the $\Phi, \omega$, and $\chi$ scan in a changing inclination angle. The diagram also shows the structural defect orientations

Generally, the size of the mosaic spreads can be evaluated from the peak widths of high resolution X-ray diffraction (HRXRD), which is characterized by the FWHM. In a previous study, the general optical and structural properties of this heterostructure were already reported [12]. The aim of the present paper is to investigate if the top InGaN/GaN multi quantum wells (MQWs) and p-GaN layer can influence the mosaic structure of the GaN buffer epi-layer. For this purpose, the detailed examination of the characterizing structural features (correlation lengths perpendicular and parallel to the substrate surface, the tilt and twist angles, and heterogeneous strain) and dislocation densities (edge and screw TDs) of the hexagonal epitaxy GaN layer is conducted. The mosaic structure is also analyzed by using a different crystal direction along those (10.l) asymmetric reflections which are the angular inclined planes to the $z-$ axis. Such properties are key issues in characterizing the structural quality of the heterostructures with a large lattice mismatch to the substrate.

\section{Experimental procedure}

The $\operatorname{In}_{x} \mathrm{Ga}_{1-x} \mathrm{~N}(x \cong 0.096)$ MQW was grown by MOCVD on a $c$-plane (00.1)-faced sapphire substrate with a $30 \mathrm{~nm}$-thick $\mathrm{GaN}$ nucleation layer. During the growth, the reactor pressure was maintained at 200 mbar. Prior to the growth of MQW, a GaN buffer layer with a thickness of $700 \mathrm{~nm}$ was deposited at $1,050{ }^{\circ} \mathrm{C}$ and then a n-type $\mathrm{GaN}: \mathrm{Si}$ layer of a thickness of $250 \mathrm{~nm}$ was grown at $1,090{ }^{\circ} \mathrm{C}$. The sample contains five periods of a 2.2-nm $\mathrm{In}_{x} \mathrm{Ga}_{1-x} \mathrm{~N}$ active layer that was grown on a thin $15.7-\mathrm{nm}$ $\mathrm{GaN}$ grown at $710{ }^{\circ} \mathrm{C}$. The cap layer was capped with a 193.6-nm-thick p-type $\mathrm{GaN}: \mathrm{Mg}$ that was grown at

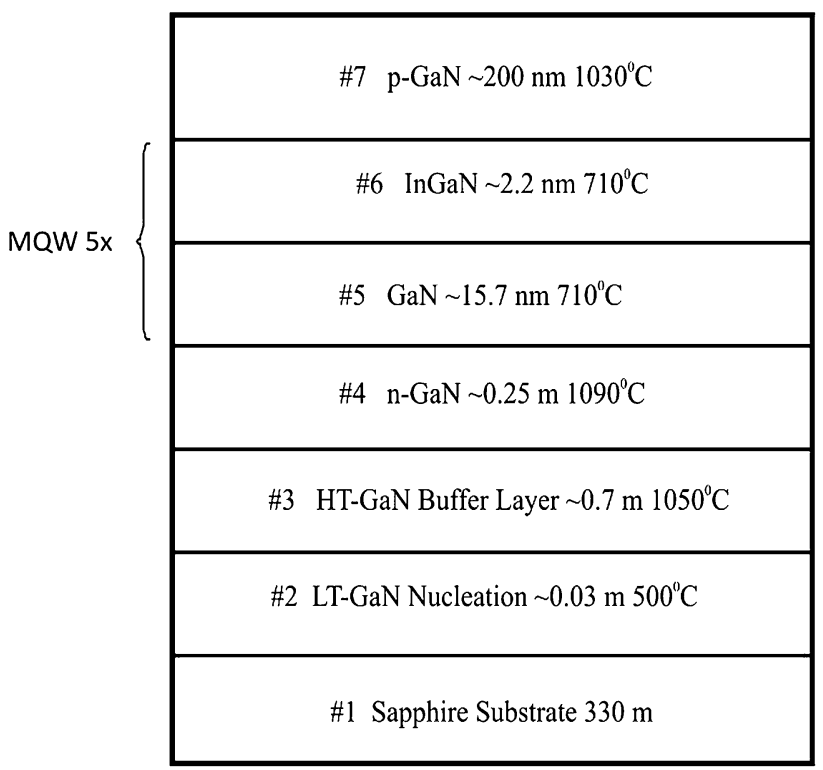

Fig. 2 The schematic cross section and growth conditions of the LED structure

$1,030{ }^{\circ} \mathrm{C}$. The schematic drawing of the MQW structure is shown in Fig. 2. The layer thicknesses and In content were measured by X-ray reflectivity and high resolution X-ray techniques in previous study [12]. At $x=0.096$ value, a strong blue emission at $2.86 \mathrm{eV}$ from photoluminescence spectrum is observed as shown in ref. [12].

The X-ray measurements were carried out on a D-8 Bruker high-resolution diffractometer using $\mathrm{CuK} \alpha_{1}$ $(1.540 \AA)$ radiation, and a 4-crystaled $\mathrm{Ge}(220)$ symmetric monochromator. Using Si calibration sample, its best resolution is 16 arcsec. For the mosaic calculations, we used the (00.2), (00.4), (00.6), (10.5), (20.4), (12.1), (10.1), (10.2) and (10.3) reflections. For the in-plane work, a vacuum holder was used to incline the sample at wide angles up to $90^{\circ}$. On the analyzed side, the reflected light passes through the horizontal divergence slit with $0.6 \mathrm{~mm}$ and a height of $8 \mathrm{~mm}$ for selecting the $\mathrm{CuK} \alpha_{1}$ radiation before the wide open scintillation detector.

\subsection{Theory of mosaic crystals}

The broadening of the symmetric or asymmetric rocking curves is the results of the tilt and twist angles and the lateral coherence length parallel to the substrate surface, as can be seen in Fig. 1. For the symmetric and asymmetric scans, the incoming and outgoing wave vectors are within the same $z$-axis containing scattering plane. In the case of the symmetric diffraction geometry, the lateral coherence length and tilt of the mosaic blocks result in the broadening of the rocking curves perpendicular to the $z$-axis [13]. The contribution of the two effects causes a 
linear dependence on the broadening of the reflection order, which is used to separate the individual contributions. For this purpose, a Williamson-Hall (W-H) plot can be used [14], which is a plot of the FWHM of the rocking curve as a function of the reflections order that is $\operatorname{FWHM}(\sin \theta) / \lambda$ plotted against $(\sin \theta) / \lambda$ for each reflection, and the plotted curve is fitted with a straight line. FWHM is the integral width of the measured profile, $\lambda$ and $\theta$ are the $\mathrm{X}$-ray wavelength and incident angle of the $\mathrm{X}$-rays, respectively. The tilt is then obtained from the slope of the linear dependence and the lateral coherence length $L_{\|}$follows from the inverse of the point of interception with the ordinate.

Another method for lateral coherence length $L_{\|}$is based on the well known pseudo-Voigt function that is provided in most graphic programs. By easily fitting this function with rocking curves, the parameter $\eta$ of the least squares and the integral width $\beta_{\omega}$ of the fit are found and then one can calculate the lateral coherence length $L_{\|}$by formulae obtained by De Keijser [15]:

$$
L_{\|}=\frac{0.9 \lambda}{\beta_{\omega}\left(0.017475+1.500484 \eta-0.534156 \eta^{2}\right) \sin \theta}
$$

For GaN grown along (0001) the plane of sapphire, the screw and edge type TDs are well known defects and are determined with the equations shown below $[16,17]$;

$N_{\text {screw }}=\frac{\mathrm{FWHM}_{(00.2)}^{2}}{9 b_{\text {screw }}^{2}}, N_{\text {edge }}=\frac{\mathrm{FWHM}_{(12.1)}^{2}}{9 b_{\text {edge }}^{2}}$

$N_{\text {dis }}=N_{\text {screw }}+N_{\text {edge }}$

where $N_{\text {screw }}$ is the screw dislocation density, $N_{\text {edge }}$ is the edge dislocation density, FWHM is measured by HRXRD rocking curves, and $b$ is the Burgers vector length $\left(b_{\text {screw }}=0.5185 \mathrm{~nm} ; b_{\text {edge }}=0.3189 \mathrm{~nm}\right)$. As it is known, the tilt range of the lattice planes for wurtzite GaN layers reflects the content of screw TDs with a Burgers vector $b=[0001]$, and the twist range of the lattice planes reflects the content of the edge-type dislocations with a Burgers vector $b=1 / 3[11 \overline{2} 0]$ [18].

If the edge type TDs are piled up in small angle grain boundaries, it is also determined by [19, 20]:

$N_{\text {edge }}=\frac{\text { FWHM }}{2.1\left|b_{\text {edge }}\right| L_{\|}}$,

where FWHM is the omega $(\omega)$-scan's peak broadening of the asymmetric (12.1) plane, determining twist angle, $b_{\text {edge }}$ is Burger vector of the edge dislocation and $L_{\|}$is the average size of the lateral coherence length. From Eq. 4, it is clearly showed that this type of edge dislocation leads to a distribution of the in-plane rotational disorder (twist) of individual crystallites resulting in a broadening of the asymmetric $\omega$-scans.

\section{Results and discussion}

Figure 3 shows the symmetric and asymmetric rocking curves together with the FWHM values for the selected (00.2), (10.2), (10.1) and (10.5) reflections. They have the sharp and narrow peaks, which are the evident for the perfect crystal surfaces of heterostructures. The same results are also possible to be observed from a Phi scan as shown in Fig. 5. The FWHMs of $\omega$ scans are obtained fitting the pseudo-Voigt 5 function [13] to the rocking curves. All rocking scans of both the $(00 . l)$ for $l=2,4$, and 6 reflections and (hk.l) reflections with either an $h$ or $k$ nonzero orientation of our sample, exhibit a FWHM of $0.0744^{\circ}-0.1312^{\circ}$.

In Fig. 3, the HRXRD symmetric (00.2) that is incorporated with an asymmetric (10.2) scan is shown as a reliable technique to characterize the crystal quality of GaN layers [8]. The defects in the structure of the sample causes significant broadening in both the symmetric (00.2) and asymmetric (10.2) rocking curve, which have fitted widths of 133 and 450 arcsec, respectively. It is noteworthy that the measured width of the (10.2) rocking curve from the sample is larger than that of the (00.2) rocking curve. This broadening of the asymmetric diffractions compared to the symmetric diffractions is an indicator of a defective structure with a large pure edge TD content, since the (00.2) peak is only broadening by screw or mixed TD while the (10.2) peak is broadened by all of the TDs.

Figure 4 shows the broadened FWHMs of $\omega$ scans with increasing $\chi$ for the sample. It can be seen in this figure that the FWHMs of $\omega$ scans increase with the increment of $\chi$. Moreover, it becomes closer when the (12.1) reflection yields at $78.6^{\circ}$ in $\chi$ as shown in Fig. 4. In fact, the angle $\chi$ reaches $90^{\circ}$ i.e., when the reflection plane is perpendicular to the surface of the sample. Additionally, it is worth pointing out that the rocking-curve widths of $\omega$ scans for this higher $\chi$ angle are close to the twist angles as shown in Table 1. Moreover, the twist angle can be obtained from FWHMs of asymmetric Phi scans versus chi angles. It gives the same result with $78.6^{\circ}$ in $\chi$. This result can be clearly seen in Fig. 4.

The diffraction geometry is shown in Fig. 2 and $\Phi$ scans are used to find the azimuth planes. Due to much thinner layers, it is impossible to realize the reflection in the lattice plane inclination of $90^{\circ}$. In Fig. 5, we would like to demonstrate that for the wurtzite structure the $\{12.1\}$-crystallography planes include 12 similar (12.1) planes. Their reflection peaks of the $\Phi$ scan do not repeat at every $30^{\circ}$. Every group of six-plane peaks, which is composed of low and high intensities, repeats at every $60^{\circ}$ as seen in Fig. 5 . Azimuth peaks with high intensity are perpendicular to the (12.1) plane but other reflection planes with low intensity are not perpendicular to (12.1) because these azimuths 
Fig. 3 The measured rocking curves of the $\omega$ scan, as well as the fitted curves using a function of pseudo-Voigt. The dotted lines are experimental data and the lines are fits to them
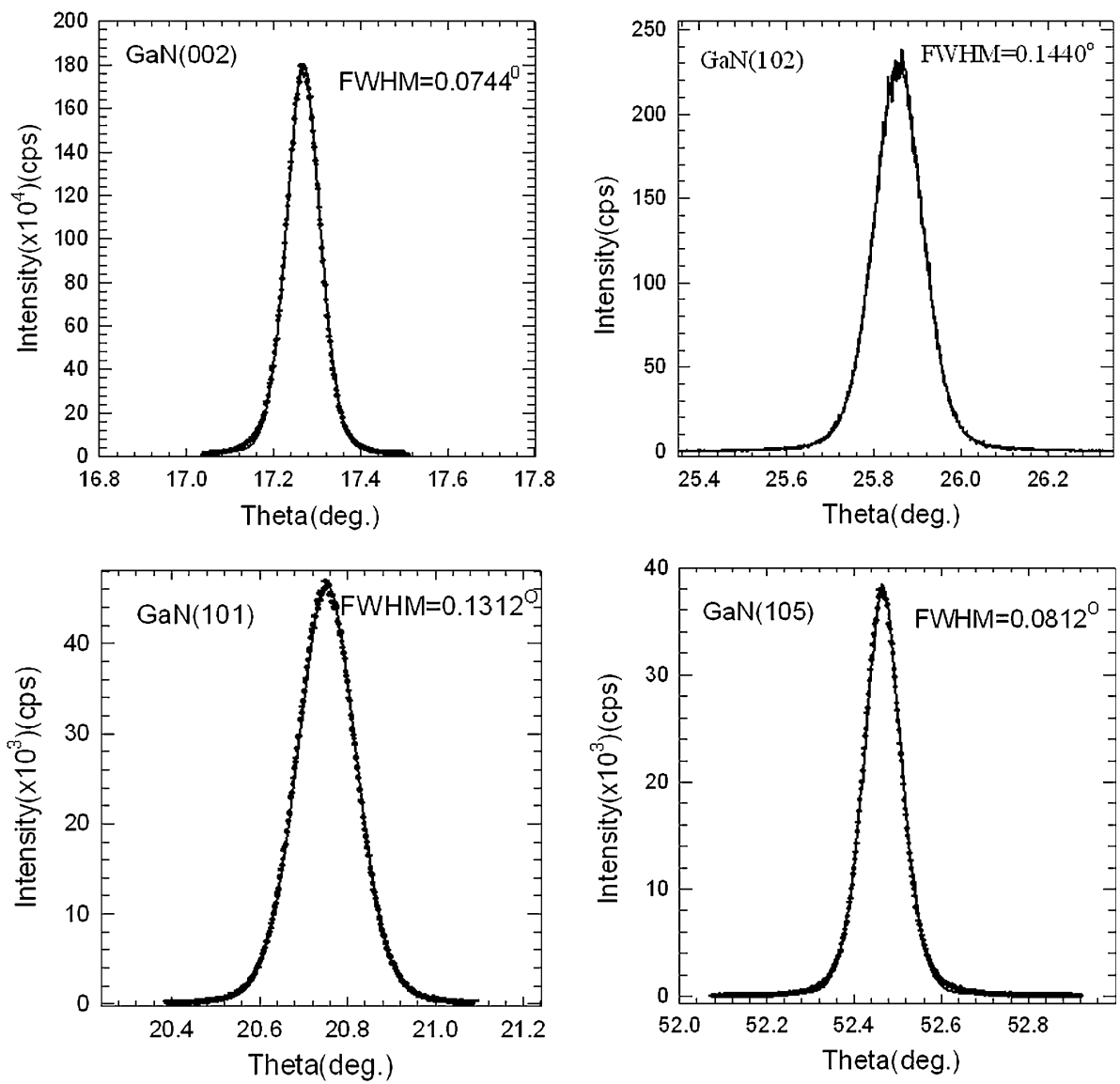

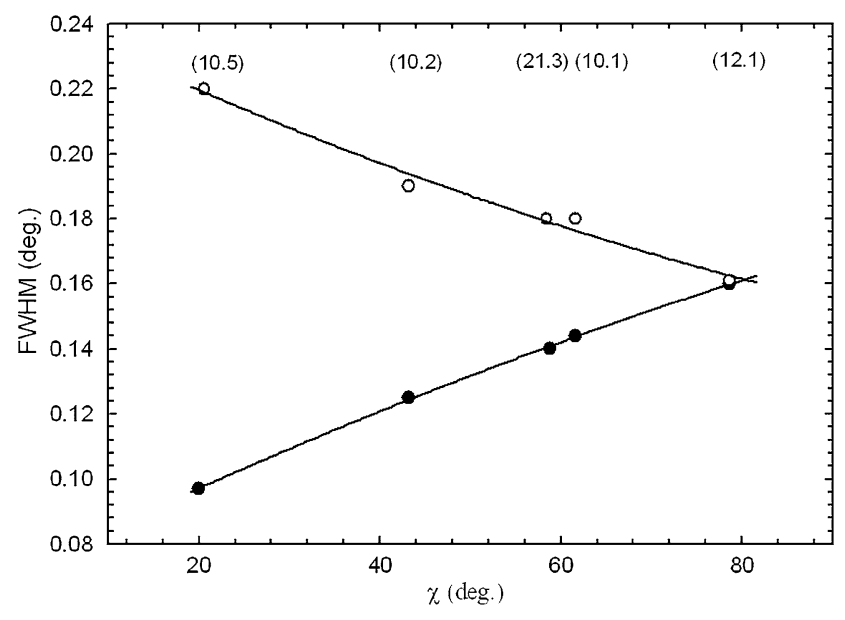

Fig. 4 Peak width of $\omega$ scans for $(h k . l)$ reflections varies with the inclination angle $\chi$ for different GaN planes. FWHMs of the $\omega$ scan increase with the increment of the $\chi$ angle. The line results from a Quadratic fit of the data

exhibit planes perpendicular to the symmetric (00.l) hexagonal InGaN layers. However, the FWHMs of $\Phi$ scans are equal within these two groups.

In Figs. 6 and 7, the W-H plots are used to calculate the size of the tilt angle, lateral coherence length, and TDs from symmetric directions. We suggest that these calculations can be made from both the symmetric and asymmetric directions. They provide an important advantage for verifying the results. In Table 1 , it is clearly shown that the results calculated from every two directions are in good agreement in a defined ratio depending on the inclination or polar angle of the sample. When the slope of the (10.l) oriented $\mathrm{W}-\mathrm{H}$ plot is compared with those of the (00.l) oriented $\mathrm{W}-\mathrm{H}$ plot, the ratio among them is 1.07. The slope values are given in the right bottom corner of every two W-H plots in Fig. 6 and 7. In comparing this ratio with the ratios in the last columns in Table 1 , it is especially reliable for the best agreement with the values of the tilt angle, lateral coherence lengths and screw type TDs. However, the edge type TDs were obtained from (12.1) in the higher chi value because this plane is affected from edge dislocation more than asymmetric other planes due to larger FWHM.

The rocking curve is plotted by rotating the sample on the $\omega$-axis. The lateral correlation length of the mosaic structure causes the peak broadening of the rocking curves as shown in Fig. 3. The peak broadening due to tilted layers is parallel to the drawn $\omega$-scan direction and the peak broadening due to a small lateral correlation length that is parallel to the surface. The lateral coherence lengths were calculated with two different techniques. One of them 
Table 1 Structural properties of MQW along all the asymmetric planes and the $10 . l(l=1,2,3$ and 5) asymmetric planes

\begin{tabular}{|c|c|c|c|c|}
\hline & $(h k . l)$ & $(10 . l)$ & AFM & Ratio $\left(x_{(h k . l)} / y_{(10 . l)}\right)$ \\
\hline \multirow{3}{*}{$D_{\text {edge }}\left(\mathrm{cm}^{-2}\right)\left(\times 10^{8}\right)$} & $6.68(\mathrm{~W}-\mathrm{H})$ & $4.74(\mathrm{~W}-\mathrm{H})$ & - & 1.41 \\
\hline & 5.28 (Eq. 4) & 3.66 (Eq. 4) & - & 1.44 \\
\hline & 8.52 (Eq. 2) & 3.13 (Eq. 2) & - & 2.72 \\
\hline$D_{\text {screw }}\left(\mathrm{cm}^{-2}\right)\left(\times 10^{7}\right)$ & 9.32 (Eq. 2) & 9.31 (Eq. 2) & - & 1.00 \\
\hline$D_{\text {dis }}\left(\mathrm{cm}^{-2}\right)\left(\times 10^{8}\right)$ & 8.94 & 4.77 & $\sim 6.00[12]$ & 1.87 \\
\hline \multirow[t]{2}{*}{$L_{\|}(\mathrm{nm})$} & $623(\mathrm{~W}-\mathrm{H})$ & $533(\mathrm{~W}-\mathrm{H})$ & - & 1.17 \\
\hline & 789 (Eq. 1) & 691 (Eq. 1) & - & 1.14 \\
\hline \multirow[t]{2}{*}{$L_{\perp}(\mathrm{nm})$} & $994^{\mathrm{a}}$ & - & - & - \\
\hline & $938(\mathrm{~W}-\mathrm{H})$ & - & - & - \\
\hline Tilt $\left(^{\circ}\right)$ & $0.069(\mathrm{~W}-\mathrm{H})$ & $0.063(\mathrm{~W}-\mathrm{H})$ & - & 1.09 \\
\hline Twist $\left({ }^{\circ}\right)$ & 0.16 & - & - & - \\
\hline \multirow[t]{2}{*}{$\varepsilon_{\perp}\left(10^{-4}\right)$} & $1.9^{\mathrm{a}}$ & - & - & - \\
\hline & $2.0(\mathrm{~W}-\mathrm{H})$ & - & - & - \\
\hline
\end{tabular}

$x$ and $y$ show the mosaic values calculated from the $(h k . l)$ and $(10 . l)$ crystal directions. DLs were calculated from the averaged value of the edge type DLs

The notations in parenthesis show the related equations and the Williams-Hall plot

${ }^{a}$ These values were calculated with the derived equations. They are explained in the text

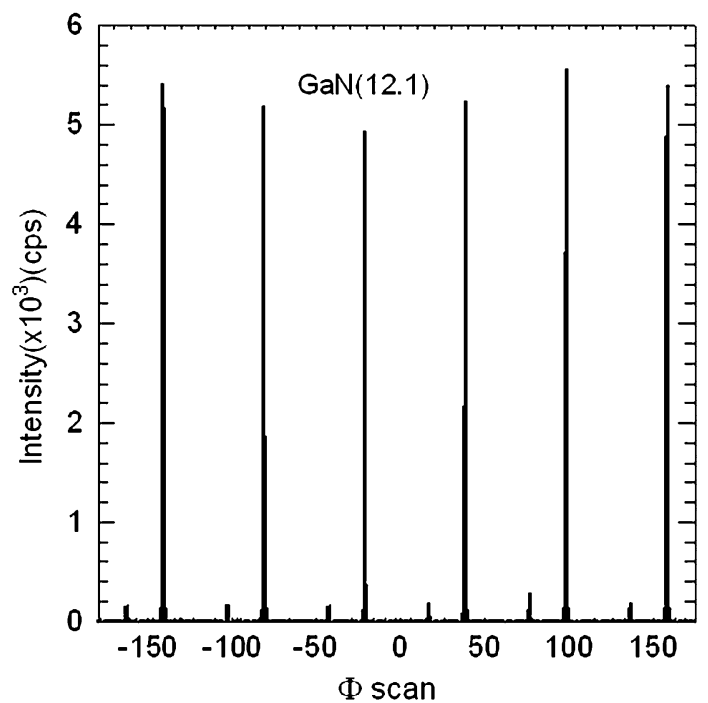

Fig. 5 Phi scan curve of asymmetric GaN (12.1) reflection plane. Every peak shows azimuths of the (12.1) plane

is the calculation shown in Eq. 1 and the other is $0.9 \lambda /\left(2 y_{0}\right)$ where $y_{0}$ is the intersection of the $\mathrm{W}-\mathrm{H}$ plot as shown in Fig. 6. Lateral coherence lengths are also obtained from the (10.l) oriented $\mathrm{W}-\mathrm{H}$ plot shown in Fig. 7. All of these values are in good agreement.

In the radial-scan direction of the symmetric reflections, a vertical coherence length normal to the substrate surface and a heterogeneous strain along the $c$-axis causes a broadening of the Bragg reflections. In the $\mathrm{W}-\mathrm{H}$ plot, $\beta_{2 \theta}(\cos \theta) / \lambda$ is plotted against $(\sin \theta) / \lambda$ for each reflection and again fitted by a straight line in Fig. 8 . From the $y$-intersection, $y_{0}$ the correlation length $L_{\perp}$ can be

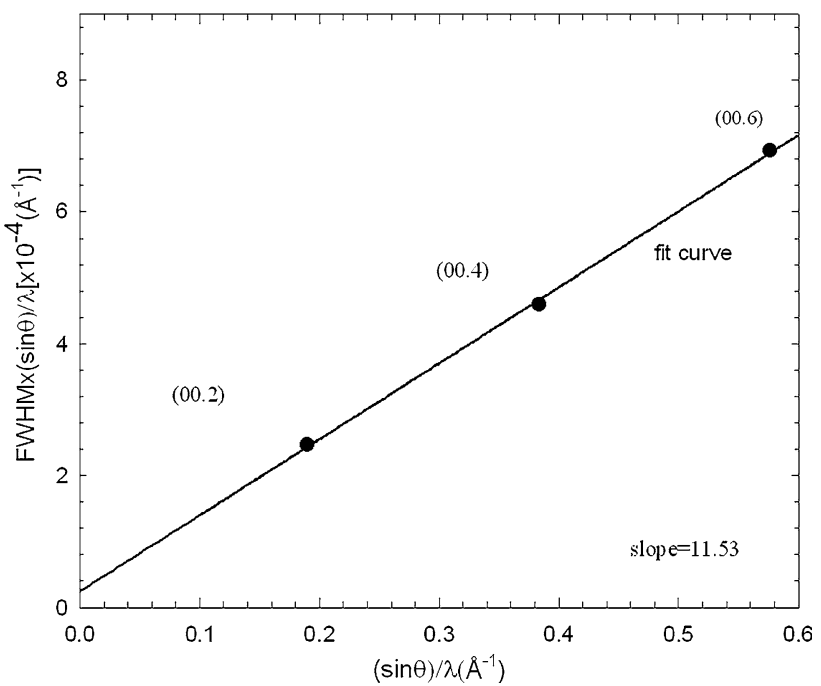

Fig. 6 Williamson-Hall plot for symmetric layers. The $\omega-2 \theta$ scans were measured for $(00 . l)$

estimated $\left(L_{\perp}=0.9 /\left(2 y_{0}\right)\right)$ and the strain $\varepsilon_{\perp}$ is obtained directly from the slope of the line, which is $4 \varepsilon_{\perp}$. Additionally, the vertical coherence length can be given with an equation found by replacing $\beta_{\omega}$ and $\sin \theta$ with $\beta_{2 \theta}$ and $\cos$ $\theta$ in Eq. 1. The vertical coherence length $L_{\perp}$ and the strain normal to the substrate surface can be calculated analytically by linking the lateral coherence lengths to that of the vertical as $L_{\perp}=L_{\|} \times\left(\beta_{2 \theta} / \beta_{\omega}\right) \times \tan \theta$ and also $\varepsilon_{\perp}=\left(\alpha_{\theta} \times \beta_{2 \theta} / \beta_{\omega}\right) /(4 \times \tan \theta)$ that was derived using the equations (correction term and heterogeneous strain equality) in ref. [13]. The $L_{\perp}$ and $\varepsilon_{\perp}$ values obtained from these terms are shown in Table 1 and compared with the 


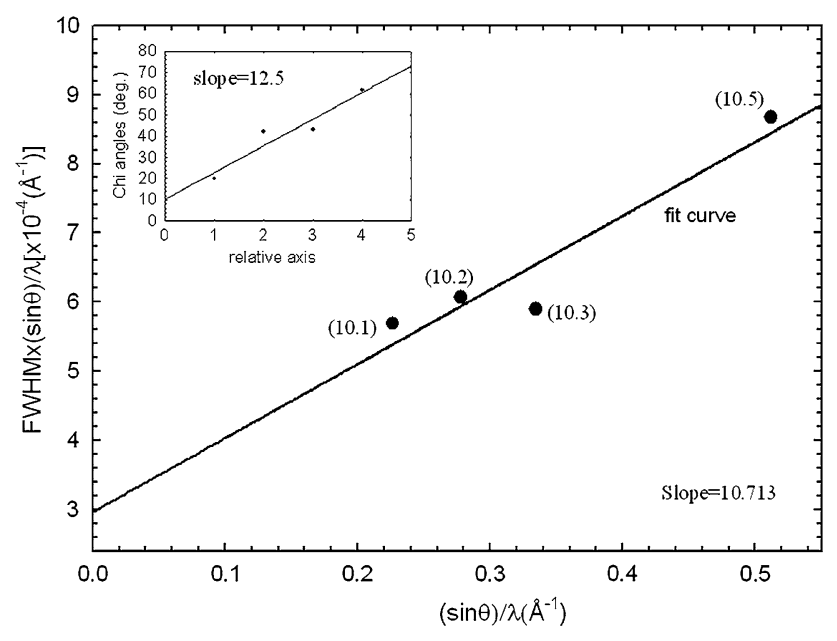

Fig. 7 Williamson-Hall plot for asymmetric layers. The $\omega-2 \theta$ scans were measured for the (10.l) reflections that are shown in the figure. The line results from a linear fit of the data

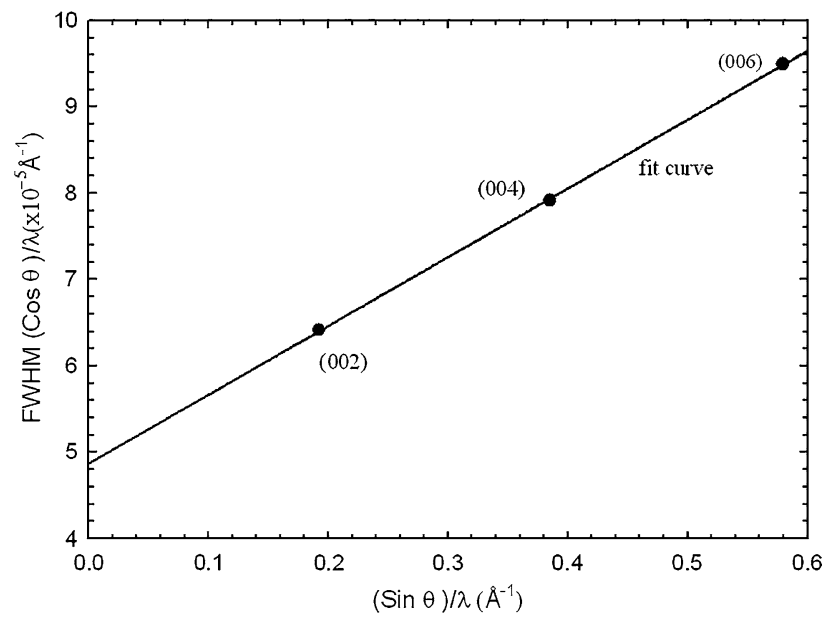

Fig. 8 Williamson-Hall plot for symmetric layers. Here FWHM is the peak broadening of $2 \theta$ scans. The line results from a linear fit of the data

values found from the $\mathrm{W}-\mathrm{H}$ curve. The results obtained from the derived equations are in good agreement with $\mathrm{W}-\mathrm{H}$ results.

For the determination of edge and screw dislocation densities from the strain broadening of asymmetric reflections, the Eqs. 2, 3, and 4 are used and the obtained values are given in Table 1. The obtained dislocation values are consistent in the same order. Table 1 shows the results for an edge dislocation density of $\sim 10^{8} \mathrm{~cm}^{-2}$ and a screw dislocation density of $\sim 10^{7} \mathrm{~cm}^{-2}$. The influence of the screw dislocation density on the peak broadening is smaller, until it reaches the order of the edge dislocation density. Therefore, the simulated broadening is very sensitive only to variations in the edge dislocation density [13]. Then, the edge and screw dislocation densities were added and the obtained DLs results were compared with the AFM result taken from the ref. [12]. They are in good agreement and in the same order as shown in Table 1. In addition, the dislocation densities of up to $10^{10} \mathrm{~cm}^{-2}$ are common in GaN-based heterostructures and high efficiency blue lightemitting diodes (LED) [11]. Here, the dislocation densities are small. For a good quality LED structure, it is evident that the tilt and twist angles are very low as shown in Table 1.

\section{Conclusion}

The defect structure of InGaN/GaN heterostructures grown in two-dimensional step-flow mode by MOCVD was investigated by using HRXRD in detail. From these measurements, the mosaic structure properties, vertical and lateral coherence lengths, TD densities, the tilt and twist angles were obtained. For these defect properties, the different directions as (00.l) and (10.l) were utilized and thereby obtained the mean rations among these directions. This can be considered as a different way to calculate the defect properties. Lateral coherence lengths and dislocation densities obtained from both the $\mathrm{W}-\mathrm{H}$ plot and dislocation equations are in good agreement and the total dislocation density is compared to that of AFM in same order. Azimuth peaks of the (12.1) oriented reflections confirm the good single crystal wurtzite hexagonal structure of the $\mathrm{GaN}$ epilayers. The obtained small tilt and twist angles show the quality of the LED structure, in which the LED has good electrical and optical properties. To conclude, the LED structure with multiple complex layers is very similar to those of the $\mathrm{GaN}$ buffer layer where the optimization of the buffer layer is crucial for obtaining a high quality LED.

Acknowledgments This work was supported by the Turkish Prime Ministry State Planning Agency under the project nos. 2001K120590 (S.Ö.) and by TUBITAK under the project nos. 104E090 and 105E066. One of the authors (Ekmel Ozbay) acknowledges partial support from the Turkish Academy of Sciences.

\section{References}

1. L. Sung-Nam, H.S. Paeka, J.K. Sona, T. Sakonga, E. Yoonb, O.H. Nama, Y. Parka, Physica B 376, 532 (2006)

2. Z. Dridi, B. Bouhafs, P. Ruterana, Semicond. Sci. Technol. 18, 850 (2003). doi:10.1088/0268-1242/18/9/307

3. X.H. Wu, L.M. Brown, D. Kapolmek, S. Keller, B. Keller, S.P. Denbaars, J.S. Speck, J. Appl. Phys. 80, 3228 (1996). doi:10. 1063/1.363264

4. N.G. Weimamn, L.F. Eastman, J. Appl. Phys. 83, 3656 (1998). doi:10.1063/1.366585

5. X. Xu, R.P. Vaudo, J. Flynn, J. Dion, G.R. Brandes, Phys. Status Solidi 202, 727 (2005). doi:10.1002/pssa.200461521

6. A.R.A. Zauner, J.L. Weyher, M. Plomp, V. Kirilyuk, I. Grzegory, W.J.P. van Enckevort, J.J. Schermer, P.R. Hageman, P.K. Larsen, J. Cryst. Growth 210, 435 (2000). doi:10.1016/S00220248(99)00886-6 
7. C.R. Miskys, M.K. Kelly, O. Ambacher, G. Martinez-Criado, M. Stutzmann, Appl. Phys. Lett. 77, 1858 (2000). doi:10.1063/ 1.1311596

8. H. Yu, M.K. Ozturk, S. Ozcelik, E. Ozbay, J. Cryst. Growth 293, 273 (2006). doi:10.1016/j.jcrysgro.2006.05.022

9. Z.T. Zhou, L.W. Guo, Z.G. Xing, G.J. Ding, J. Zhang, M.Z. Peng, H.Q. Jia, H. Chen, J.M. Zhou, Chin. Phys. Lett. 24, 1641 (2007). doi: $10.1088 / 0256-307 X / 24 / 6 / 058$

10. Y. Narukawa, Y. Kawakami, M. Funato, S. Fujita, S. Nakamura, Appl. Phys. Lett. 70, 981 (1997). doi:10.1063/1.118455

11. N.A. El-Masry, E.L. Piner, S.X. Liu, S.M. Bedair, Appl. Phys. Lett. 72, 40 (1998). doi:10.1063/1.120639

12. S. Korcak, M.K. Ozturk, S. Corekci, B. Akaoglu, H. Yu, M. Cakmak, S. Saglam, S. Ozcelik, E. Ozbay, Surf. Sci. 601, 3892 (2007). doi:10.1016/j.susc.2007.04.088

13. T. Metzger, R. Hopler, E. Born, O. Ambacher, M. Stutzma, R. Stommer, M. Schuster, H. Gobe, S. Christiansen, M. Albrecht, H.P. Strun, Philos. Mag. A 77, 1013 (1998)
14. G.K. Williamson, W.H. Hall, Acta Metall. 1, 22 (1953). doi: 10.1016/0001-6160(53)90006-6

15. T.H. De Keijser, E.J. Mittemeijer, H.C.F. Rozendaal, J. Appl. Cryst. 16, 309 (1983). doi:10.1107/S0021889883010493

16. R. Gay, P.B. Hirsch, A. Kelly, Acta Metall. 1, 315 (1953). doi: 10.1016/0001-6160(53)90106-0

17. T. Ide, M. Shimizu, X.Q. Shen, K. Jeganathan, H. Okumura, T. Nemoto, J. Cryst. Growth 245, 15 (2002). doi:10.1016/S00220248(02)01665-2

18. X.H. Zheng, H. Chen, Z.B. Yan, Y.J. Han, H.B. Yu, D.S. Li, Q. Huang, J.M. Zhou, J. Cryst. Growth 255, 63 (2003). doi:10.1016/ S0022-0248(03)01211-9

19. P.F. Fewster, J. Appl. Cryst. 22, 64 (1989). doi:10.1107/S0021 889888011392

20. M.J. Hordon, B.L. Averbach, Acta Metall. 9, 237 (1961). doi:10. 1016/0001-6160(61)90073-6 